\author{
William J Cobb* \\ National University of Medical Sciences, University of Osteopathy, USA
}

Submission: July 15,2018; Published: October 23, 2018

*Corresponding author: William JCobb, National University of Medical Sciences, University of Osteopathy, USA, Tel: 5865494552;

Email: kineticrehab@mail.com

Abstract

Alzheimer's disease (ADZ) is a devastating one that not only has a profound cost on the life of the sufferer but also upon the families, caregivers and the medical economy surrounding disease management. ADZ affects a vast human population across Northern America and indeed the world and is listed as being the 6th leading cause of death in the United States (USA).It is estimated that by 2050, there will be as many as 16 million Americans living with ADZ [1,2], and the disease progression is on a steadily increasing trajectory with an estimated new diagnosis being made every 66 seconds in the United States alone. The typical survival rate post prognosis is estimated to be between 4 to 8 years[1,2].

There has been an 89\% increase in deaths due to ADZ between 2000 and 2014 and it is further estimated that 1 in 3 seniors will die with some form of dementia. Nationally, ADZ affects 5.5 million Americans and has been shown to have a substantial financial cost both nationally and indeed globally. According to recent research conducted in 2016, 15.9 million family caregivers provided an estimated 18.2 billion hours and $\$ 230$ billion to people with dementia. With more recent research showing that in 2017, ADZ cost the United States alone $\$ 259$ billion [1,2], it is estimated that by 2050 the costs associated with dementia could be as much as $\$ 1.1$ trillion [1,2]. From a global perspective, the cost of ADZ and dementia is estimated to be around $\$ 605$ billion.Currently, ADZ is the only disease in the 10 leading causes of deaths in the United States that cannot be cured, prevented or slowed. It is estimated that around 1 in 10 Americans within the age bracket of 65 lives with ADZ. The future projections for disease manifestation are bleak with a projection that a $14 \%$ rise will be seen in every state across America between 2017 and 2025 [1,2]. It is currently estimated that more than 5 million Americans are currently living with ADZ, and ppopulationswithin the age range of over 85 are estimated to make up one-third of sufferers. The statistical proportion of the population with ADZ in the United States is vast, with the age group of 75-84 making the most significant percentile at $44 \%$. Populations in the age range of 85 and above make up a $38 \%$ percentile with age ranges of 65-74 making up a $15 \%$ percentile and the age rangeof under 65 s making up a $4 \%$ percentile.

Mercury is arguably one of the most toxic substances known to human health. Mercury toxicity has been shown to have almost identical biochemical similarities to that of ADZ as seen in brain tissue studies. Mercury is a naturally occurring element within diet and is at increased levels in diets rich in certain fish and shellfish [3- 5]. Modern medical science has been attempting for many years to link the dietary impact of mercury with that of neurological pathological change with varying results. It is currently understood that mercury at certain levels will ultimately destroy brain tissue, however mercury has been proven to be extensive in certain fish and shellfish which also have important dietary values. This is somewhat of a dichotomy, as it has been suggested that a large contributor to the progression of the dementias is due to lack of protein synthesis due to inadequate nutritional intake. The levels at which mercury is deemed toxic in the human varies from text to text with pharmacological chelation being the chosen medical course of treatment at acutely toxic levels only [3-5].

Over the last few years, there has been an array of hypotheses surrounding ADZ brought to the forefront of scientific research. These have included the: Neurovascular hypothesis (Nh), Tau hypothesis (Th) Amyloid hypothesis (Ah) and the Cholinergic hypothesis (Ch). For the purpose of this work, the Tau and Neurovascular hypothesis will be the only two studied in reference to ADZ progression with consideration of methylmercury toxicity. The Tau Hypothesis (Th) proposes that the tau protein abnormalities initiate ADZ disease progression via hyperphosphorylation of the tau protein with other threads of tau, culminating in the formation of neurofibrillary tangles inside nerve cell bodies, resulting in the destruction of the microtubules and leading to the destruction of the cell's cytoskeleton [3-5].

The neurovascular hypothesis (Nh) proposes that poor functioning of the blood-brain barrier may be involved in ADZ progression [3-5]. Both of the hypotheses suggest that ultimately the result of either abnormal protein distribution and or vascular responses end with the destruction of brain tissue consistent with that of ADZ. How and why these processes take place and the factors that may increase these responses is a hot topic of medical science. Some of the factors that have been considered in relation to the progressive nature of ADZ are ethnic, gender and dietary links. The common thread that appears to be seen across populations most impacted by ADZ is that of a dietary linkage [5,6]. 


\section{Anatomy Physiology \& biochemistry international journal}

\section{Aim of Research Review}

The aims of this research review are to consider:

a. The biochemical interaction of ADZ progression in line with that of Methylmercury Toxicity, whilst considering the unique similarities of biochemical protein mis-folding using the TAU hypothesis to consider both ADZ and Methylmercury Toxicity (MT).

b. The biochemical interaction of ADZ progression in line with that of Methylmercury Toxicity, whilst considering the unique similarities of biochemical protein mis-folding using the Neurovascular hypothesis to consider both ADZ and Methylmercury Toxicity (MT).

c. The relationship of Methylmercury Toxicity and that of chelation agents of both artificial (pharmacological) and naturally occurring agents for the purpose of biochemical comparison on chelation effectiveness on toxicity.

d. The pharmacological and naturally occurring chelating agents comparatively for the purpose of consideration of potential dietary links with ADZ progression whilst considering the depletion of trace metals via theprocess of chelation.

\section{Objectives of Research Review}

The objectives of this research review are to:

a. Further review and enhance current literature and research surrounding both pharmacological and naturally occurring chelation for Methylmercury Toxicity, whilst linking this with ADZ progression for the purpose of dietary considerations for disease progression and or regression.

b. Further enhance research into potential dietary links to chelation and indeed ADZ progression through Methylmercury Toxicity.

c. Further identify common factors in Methylmercury Toxicity and ADZ progression in relation to potential naturally occurring dietary sources.

\section{Methylmercury Toxicity}

Mercury is one of the most toxic substances known to human beings. Several lines of research have suggested that the link between mercury toxicity and progressive ADZ follows a predictable trend with reference to the biochemical progressive nature of the disease. Some autopsy studies have found increased mercury levels in the brain tissues of ADZ patients with measurements of mercury levels in blood, urine, hair, nails, and cerebrospinal having mixed value outcome results overall. In vitro models (animal models) have shown that inorganic mercury reproduces all of the pathological changes as seen in ADZ [3-5]. Significant publications and research support the initial theory that mercury exposure causes significant biochemical changes to the human brain which exhibits hallmark signs consistent with that of progressive ADZ symptomology from a "biochemical perspective". The rationale for having an answer as to the steps preceding ADZ are important in order to gain an understanding of the biochemical processes involved in mercury toxicity. The biochemical steps of normal and abnormal physiological processing are absolutely essential in order to unwind the steps for a potential reversal process. This being said, at this current time, the application of being able to unwind the Tau protein mis-fold is not possible, however, additional understanding could further manage and identify potential risk factors of ADZ.

The biochemical process by which mercury impacts the human brain starts with the destructive nature of mercury on the biochemical pathways. Firstly, mercury rapidly inhibits thiolsensitive enzymes like tubulin, creatine kinase and glutamine synthetase and overly dramatically affects the metabolism and axonal structure [3-5].The biochemical reaction of mercury upon brain tissue continues with the stripping of tubulin leading to the formation of NFTs and the exposing Tau for hyper-phosphorylation followed by elevated production of $\beta$ - amyloid protein that can aggregate into senile plaques [5]. The aforementioned reaction is the most widely accepted diagnostic pathological hallmarks for $\mathrm{ADZ}$ and is consistent with the modus operandi as seen in mercury toxicity. In essence, what is understood about mercury toxicity and $\mathrm{ADZ}$ is that there is a breakdown in the normal biochemical pathways that leads to an abnormal or pathological change, and it is this breakdown event that ultimately has an instigating factor be this diet, environment or something else.

The hypothesis for ADZ that NFTs, hyper-phosphorylated Tau, amyloid plaques and increased oxidative stress observations are the result of neuronal mercury toxicity. [3-5], means that the biochemical process as detectable in mercury toxicity is not the cause of ADZ but rather the result of its toxicant effects on the biochemical processes in the brain. Mercury, in its elemental and organic forms has been suggested as the most likely ubiquitous environmental toxicant that affects the brain enzymes, which in turn leads to the most characteristic clinical signs and markers consistent with ADZ [3-5]. In essence, it is the argument from many lines of research that the end result is that mercury toxicity is very similar to that of the biochemical pathological changes of brain tissue as seen in ADZ and indeed vice versa [3-5]. General public, as fish and shellfish are an excellent source of quality protein and other essential nutrients being low in saturated fat and containing omega- 3 fatty acids.

\section{Naturally Occurring Mercury}

In the Diet: Interestingly there is a high prevalence of Mercury within the food chain and indeed it is often a surprise to learn that nearly all fish and shellfish contain traces of it. This is an interesting and often confusing contradiction to the general public, as fish and shellfish are an excellent source of quality protein and other essential nutrients being low in saturated fat and containing omega-3 fatty acids.The Food and Drug Administration (FDA) has 
however put forward public health warnings outlining that nearly all fish and shellfish contain traces of mercury and that for most people, the risk from mercury through eating fish and shellfish is not a health concern. Yet, some fish and shellfish contain higher levels of mercury that may be harmful to the unborn or young children's developing nervous system. The risks from mercury in fish and shellfish depend on the amount of fish and shellfish eaten and the levels of mercury in the fish and shellfish themselves. Therefore, the FDA and the Environmental Protection Agency (EPA) have advised women who may become pregnant, pregnant women, nursing mothers and young children to avoid some types of fish and eat fish and shellfish that are lower in mercury $[5,6]$. Although fish and shellfish have the highest in actual Mercury content, corn syrup and rice has also been shown to be a contributor mainly due to the manufacturing and harvesting process $[5,6]$. Methylmercury toxicity in general medical practice is medically managed by the use of chelators to remove heavy toxic metals from the body. The use of chelating agents is not a commonly used methodology, bar serious risk to life, due to the side effects of chelation upon the human [8-10](Figure 1).

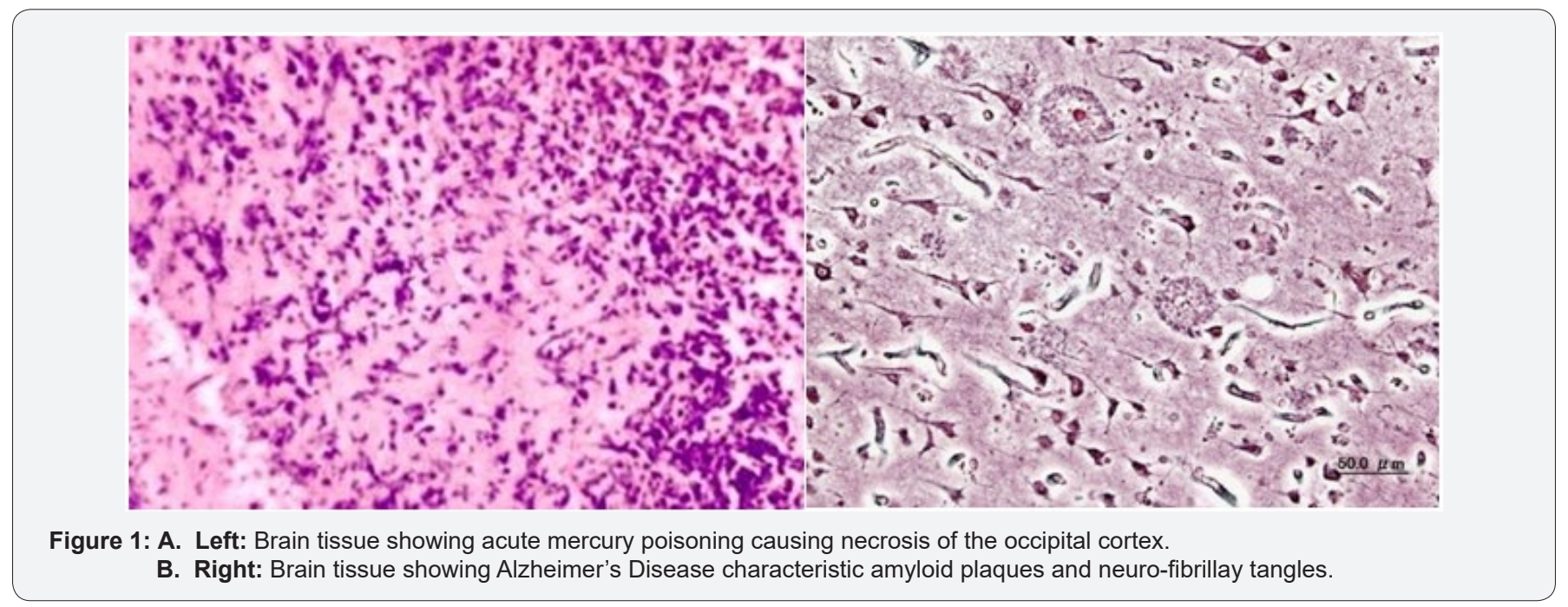

\section{Chelators}

Chelators are organic chemicals that bond with metal ions to produce a chelate compound.The process of removal of heavy metals from the body is known as chelation. The word chelation originates from the Greek name for "Claw" as early Greek physicians believed the certain substances held onto heavy metals and drew them out of the body via the digestive system. Scientifically, there is a divide as to the effectiveness of non-pharmacological chelators and that of naturally occurring chelators [8-10].Some research has suggested that iron-chelation therapy may be an effective therapy for treating iron-overload disorders and that iron chelators may have additional utility in the treatment of a variety of conditions related to oxidant stress, ranging from cardiovascular to neurodegenerative to inflammatory to malignant disease [8-10]. Chelating with pharmacologically developed drugs is indicated primarily for acute poisonings by some metals, especially lead, arsenic, mercury, and iron [9,10]. From a western medical standpoint, in cases of acute metal poisoning, although chelation agents may have dangerous side effects, the risks are considered worthwhile in the face of toxicity which may be fatal or cause serious, even permanent injury to the sufferer [8-10].

For the purpose of this work, pharmaceutical chelators will refer to FDA approved agents that are used in current medical practice with non-pharmaceutical chelators referring to naturally occurring chelating agents as seen in diet and supplements. The major difference between approved pharmaceutical and nonpharmaceutical chelators for heavy metal toxicity is essentially that within current medical practice in the United States, drugs that are used for activities such as chelation are rigorously tested and peer reviewed,and in turn are glossaried within pharmaceutical texts as opposed to non-pharmaceutical chelators which come in many forms such as from dietary intake and indeed supplements and fall under that of non-pharma. The nonpharma derived chelators are not defined in the way in which the aforementioned pharmacological agents are. This leaves somewhat of a void with reference to the potentiality of botanical medical agents and the consideration for such agents in treatment. From a pharmacological perspective, there are several approved and reviewed chelating drugs that are used in the United States healthcare system. These include, succimer, dimercaprol (BAL), edetate calcium disodium, deferoxamine and penicillamine [8$10]$.

Although it could be argued that many naturally occurring chelators mimic that of pharmacologically derived agents from a chemical perspective, it is beyond the scope of this work to integrate these comparative chemical analytical studies. Within current medical practice, chelating pharmacologicals are only given for diagnosed metal toxicity because of the suggested serious side effects, one of which being that of non-specific binding, in which agents bind even essential "trace" metals in the body, such as copper, zinc and calcium [11-13]. 
Due to pharmacologically derived chelators having the adverse side effect of depleting trace metals, they are only indicated primarily for acute poisonings. In comparison, naturally occurring chelators which are found in amino acids, obtained from proteins such as meats, fish and eggs have a resultant chelation effect which is without the sudden and diffuse trace mineral depletion [29-31]. Pharmacologically derived chelating agents may indeed remove the offending heavy metal whilst also removing other important trace elements along its path. In opposition, naturally occurring agents may have the ability to remove heavy metals in a less destructive manner but in a slower time frame. This is an interesting point of discussion as to when and how and what quantity of chelation may be needed to manage a heavy metal toxicity crisis. This is a point that should also be considered with reference to pathological disease progression in line with methylmercury toxicity and indeed comparatively with $\mathrm{ADZ}$, as the progressive nature of toxicity will inevitably lead to pathological changes in brain tissue [11-13].

The progressive nature of ADZ has been researched extensively in relation to dietary correlations. Lines of research have shown that protein deprivation decreases the liver content of several enzymes that the body needs to remove toxins which has also been seen in trans fats, that in turn interfere with the detoxification enzyme system [11-13]. Lack of protein intake is another area within dementia research that has shown that during the progression of the disease, protein intake becomes depleted as the patient forgets to eat and or eats food products that may further increase the disease processes [32].

According to numerous authors [14-17], besides amino acids, other organic acids found in the body or in foods can act as chelating agents, including: acetic acid, citric acid, ascorbic acid, lactic acid and natural fats. The high percentages of ADZ occurrence as seen in the Unites States dwarfs other counties such as Singapore which has a fatality rate of $0.19 \%$ per 100.000 of a standardized age range in comparison to the United States rate of $45.58 \%$ per 100.000 [18]. The dietary differences between the United States and that of Singapore has been considered for many years in research looking at dietary impacts of heart disease and type 2 diabetes being linked directly to dietary factors of the Western culture/diet. The United States has over the past century dramatically decreased essential nutrient deficiencies, but has seen a rise in non-communicable diseases, specifically chronic diet-related diseases. The United States has developed a history of poor eating and physical activity patterns, which has ultimately culminated in dietary related diseases such as cardiovascular disease, high blood pressure, type 2 diabetes and some cancers [8].

About half of all-American adults-117 million individualshave one or more preventable chronic diseases, many of which are related to poor quality eating patterns and physical inactivity with more than two-thirds of adults and nearly one-third of children and youth being overweight or obese [8]. With the dietary link of diet and disease in mind, one must consider the application of diet upon biochemical processing overall and in particular for this work, with that of ADZ progression. Dietary demographics may play a pivotal role in the radically reduced rates of progression between the United States and that of other countries such as Singapore. It may be a point to consider that, although certain fish and shellfish contain greater levels of mercury content, they also contain essential fatty acids and are a rich protein resource that is needed to promote neuro-physiological processing through normal biochemical pathway processing $[20,21]$. Research is now more than ever asking the question whether diet could play a substantial role in ADZ progression as well as many other dementias when considering the population demographics between East and West.

\section{Biochemical Reactions within Alzheimer's Disease}

ADZ is a very well-researched area within modern medical science and indeed is one in which a great deal of different methodologies of research are being considered. The disease itself has been suggested to be a possible protein mis-folding disease [19-21].Protein mis-folding and protein mis-folding diseases in general are clinically described as proteopathies which refers to a class of diseases in which certain proteins become structurally abnormal [19-21]. This abnormal behavior of the protein disrupts the function of cells, tissues and organs of the body and the proteins that fail to fold into their normal configuration may become non-functional and or become a toxicologic instigator/s for disease propagation [20-23]. There is an array of protein conformational disorders, or protein mis-folding diseases which include: Creutzfeldt-Jakob disease and other prion diseases, Alzheimer's disease, Parkinson's disease, amyloidosis, and a wide range of other disorders [19-23].

ADZ is considered a protein mis-folding disease due to the accumulation of abnormally folded amyloid beta protein found in the brains of ADZ patients. Amyloid beta $(A \beta)$, is a short peptide that is an abnormal proteolytic by-product of the transmembrane protein amyloid precursor protein (APP). Functionally, it is not clear the roleAPP plays, however it is thought to involved in neuronal development[19-23].

\section{Presenilins}

Presenilin's are the components of the proteolytic complex which is involved in APP processing and degradation[24-26]. Amyloid beta monomers are soluble and contain short regions of beta sheet and polyproline II helix secondary structures in solution and structurally are largely alpha helical in membranes. At sufficiently high concentrations, they undergo a conformational change to form a beta sheet rich tertiary structure that aggregates to form amyloid fibrils. These in turn deposit outside neurons in dense formations known as senile plaques or neuritic plaques in less dense aggregates as diffuse plaques, and sometimes in the walls of small blood vessels in the brain in a process called amyloid angiopathy or congophilic angiopathy [24-26]. 


\section{Aggregation of the Tau Protein}

The tau protein is a microtubule-associated protein expressed in neurons that normally acts to stabilize microtubules in the cell cytoskeleton. Like most microtubule-associated proteins, tau is normally regulated by phosphorylation [24-26]. Due to the hyperphosphorylated tau which accumulates as paired helical filaments that in turn aggregate into masses inside nerve cell bodies known as neurofibrillary tangles and as dystrophic neurites associated with amyloid plaques, ADZ is thus also considered a tauopathy due to its abnormal aggregation [24-26]. Although little is known about the process of filament assembly, it has recently been shown that a depletion of a prolyl isomerase protein in the parvulin family accelerates the accumulation of abnormal tau [24-26].

\section{Neuro-inflammation}

The process of neuro-inflammationis also involved in the complex cascade leading to ADZ pathology and symptoms. Considerable pathological and clinical evidence documents immunological changes associated with ADZ, including increased pro-inflammatory cytokine concentrations in the blood and cerebrospinal fluid. Whether these changes may be a cause or consequence of ADZ remains to be fully understood, but inflammation within the brain, including increased reactivity of the resident microglia towards amyloid deposits, has been implicated in the pathogenesis and progression of ADZ [24-26].

\section{The Tau Hypothesis of ADZ Progression}

The initial hypothesis that will be considered in this work is that of the Tau Hypothesis. The Tau hypothesis has long been suggested as the main step in ADZ due to the observation that the deposition of amyloid plaques does not correlate well with neuron loss $[27,28]$. The hypothesis promotes that a mechanism for neurotoxicity surrounds the loss of the microtubule-stabilizing tau protein which leads to the degradation of the cytoskeleton. There has not been a uniform agreement on whether tau hyperphosphorylation precedes or is caused by the formation of the abnormal helical filament aggregates [27,28]. Proponents for the hypothesis also suggest other diseases known as tauopathies link the commonality of the same protein mis-folding pattern. In contrast, a degree of research has suggested that amyloid is the primary causative agent as seen in ADZ $[27,28]$. The unfortunate overall outcome of ADZ is the destruction of brain tissue resulting in loss of function and the inevitable death of the sufferer. The biochemical process to the point in which ADZ advances is and will continue to be an ongoing topic of research until an instigating factor (from a biochemical perspective) can be identified(Figure 2).

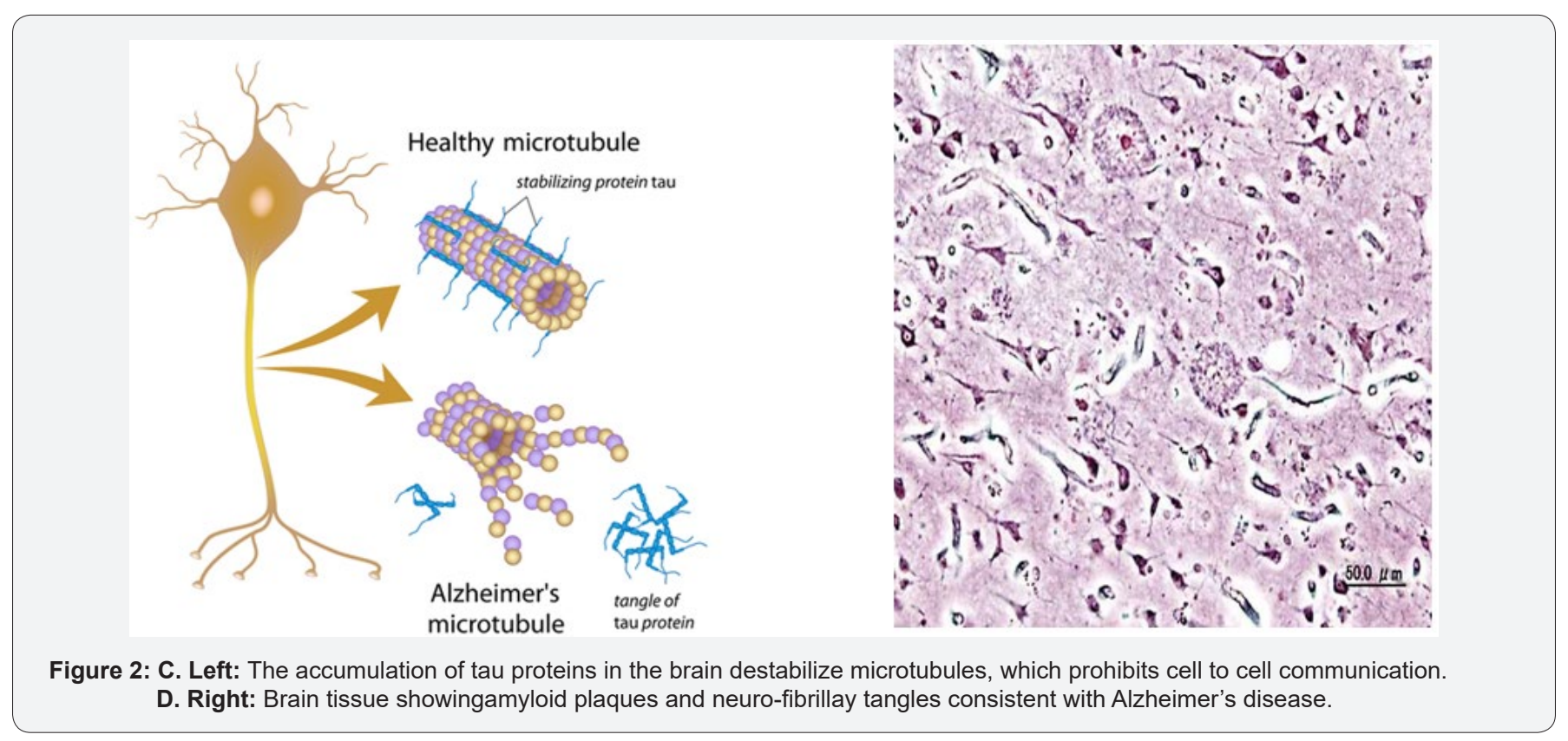

\section{The Vascular Hypothesis of ADZ Progression}

The vascular hypothesis of ADZ has previously considered risk factors that may contribute to cerebral vascular profusion. The idea that vascular dementia and ADZ may be linked with cardiovascular disease and carotid artery atherosclerosis has been suggested due to the hypothesis that it may exert pathological changes by chronically lowering cerebral hypo-perfusion during ageing[33-35].

\section{Current Research Findings}

Morriset al.[22], conducted research looking at the Association of Seafood Consumption, Brain Mercury Levels, and APOE $\varepsilon 4$ Status with Brain Neuropathology in Older Adults.

\section{Method}

The methodology used by Morriset al.[22]was a crosssectional analysis of deceased participants in the Memory and Aging Project clinical neuropathological cohort study, 2004- 
2013. The participants of the study resided in Chicago retirement communities and subsidized housing. The study included 286 autopsied brains of 554 deceased participants (51.6\%). The mean (SD) age at death was 89.9 (6.1) years, 67\% (193) were women, and the mean (SD) educational attainment was 14.6 (2.7) years.

\section{Outcomes and Measurements}

The main outcomes and measurements of the research looked for dementia-related pathologies, Alzheimer disease, Lewy bodies, and the number of macro-infarcts and micro-infarcts. Dietary consumption of seafood and n-3 fatty acids was annually assessed by a food frequency questionnaire in the years before death. Tissue concentrations of mercury and selenium were measured using instrumental neutron activation analyses.

\section{Results of Research}

The results showed that among the 286 autopsied brains of 544 participants, brain mercury levels positively correlated with the number of seafood meals consumed per week $(\rho=0.16$; $\mathrm{P}=$ .02). In models adjusted for age, sex, education, and total energy intake, seafood consumption ( $\geq 1$ meal[s]/week) was significantly correlated with less Alzheimer disease pathology including lower density of neuritic plaques $(\beta=-0.69$ score units [ $95 \% \mathrm{CI},-1.34$ to -0.04$])$, less severe and widespread neurofibrillary tangles $(\beta=$ -0.77 score units [ $95 \% \mathrm{CI},-1.52$ to -0.02$]$ ), and lower neuropathologically defined Alzheimer disease $(\beta=-0.53$ score units [95\% CI, -0.96 to -0.10$]$ ) but only among apolipoprotein E (APOE $\varepsilon 4)$ carriers. Higher intake levels of $\alpha$-linolenic acid (18:3 n-3) were correlated with lower odds of cerebral macro-infarctions (odds ratio for textiles 3 vs 1, 0.51 [95\% CI, 0.27 to 0.94]). Fish oil supplementation had no statistically significant correlation with any neuro-pathologic marker. Higher brain concentrations of mercury were not significantly correlated with increased levels of brain neuropathology.

\section{Researchers Conclusion}

The study concluded that in a cross-sectional analysis, moderate seafood consumption was correlated with less Alzheimer disease neuropathology. It was also suggested that seafood consumption correlated directly with higher brain levels of mercury although the levels were not correlated with brain neuropathology.

\section{Relevance of Study}

The research of Morriset al. [22]raises an interesting point in relation to the reduction of ALZ comparatively with dietary factors. The point that is somewhat of a contradiction is the level of mercury found in brain tissue. Methylmercury toxicity has as previously discussed been shown to mimic that of ALZs pathophysiological hallmark. One could consider that from a chelating consideration, this study does not show a positive metal removal from a non-pharma approach but does show that of a positive correlation of ALZ regression from a non-pharma approach (dietary) whilst actually increasing metal toxicity.

\section{Current Research Findings}

Fung et al.[23]considered trace element neurotoxicity as an etiologic factor for ADZ. The research looked at the concentrations of mercury in seven different brain regions from deceased patients histologically confirmed with ADZ or multiple sclerosis as compared to control subjects without known central nervous system and renal disorders. The research acknowledged that brain mercury concentrations in the deceased subjects could have arisen from amalgam restorations, diet, and the working environment.

\section{Method}

The methodology used by Fung et al.[23] was to use frozen autopsy specimens from a control group of ADZ and multiple sclerosis MS patients. The research focused on seven regions of the brain including the frontal cortex, temporal cortex, occipital cortex, putamen, hippocampus, corona radiata and corpus callosum. Concentrations of selenium using instrumental neutron activation analysis and mercury using radiochemical neutron activation analysis were performed to ascertain levels.

\section{Results of Research}

The results found that concentrations of mercury and the mercury/selenium molar ratios were significantly lower in the hippocampi of multiple sclerosis patients as compared to agedmatched controls. However, no statistically significant differences were detected for the concentrations of mercury and the mercury/ selenium molar ratios for the remaining six brain regions among these groups.

\section{Researcher's Conclusion}

The conclusions of the research suggested that since brain mercury concentrations from deceased subjects with either Alzheimer's disease or multiple sclerosis are not significantly higher than controls, there is no scientific support that mercury plays a significant role in the pathogenesis of the aforementioned neurologic disorders. The researcher suggested that there is not a significant increase although the measurements have been shown to have some potential problems within averaging of mercury content across large brain areas. The researcher also suggested that some of the autopsy studies have potential problems such as gross averaging of mercury content across large brain areas, long lags between autopsy and measurement and not taking into account the volatile nature of $\mathrm{Hg}$. The overall theme of the research suggested that the autopsy methodology for such a study would need to address procurement and testing strategies in order to ascertain more conclusive data. The autopsy methodology of Fung et al.[23] compared with the later work of Morris et al.[22] seems to lay a foundational concept for autopsy studies which need to address some of the fundamental sampling problems.

\section{Biochemical Reactions within Methylmercury Toxicity}

Mercury ions produce toxic effects by protein precipitation, enzyme inhibition, and generalized corrosive action [36-38]. The 
destructive nature of mercury on human brain tissue is due to its binding effects. Mercury not only binds to sulfhydryl groups but also to phosphoryl, carboxyl, amide, and amine groups. [36-38]. This is a further problem due to proteins (including enzymes) being highly susceptible to mercury. When bound to mercury, most proteins are rendered inactive. Toxicity can be considered as in part related to the oxidative state and to the chemical form (organic versus inorganic) [36-38].

As mentioned previously, the main route of exposure to methylmercury is through the ingestion of fish. Most fish, both freshwater and saltwater, contain methylmercury at significant levels over other dietary groups [36-38] within methylmercury toxicity; the gastrointestinal tract is the primary route of absorption with absorption through the skin and the lungs being alternative routes. When in the circulation,methylmercury enters erythrocytes where more than $90 \%$ will be found bound to hemoglobin with smaller amounts being bound to plasma proteins. Interestingly, only around $10 \%$ of the methylmercury is found in brain tissue. Once in brain tissue, methylmercury slowly undergoes demethylation to an inorganic mercuric form $[36,37]$. The problem of toxicity is further exacerbated by the fact that methylmercury readily crosses the placenta to the fetus, where deposition within the developing fetal brain can occur causing catastrophic abnormalities. This is due to the methylmercury causing focal necrosis of neurons and destruction of glial cells ultimately affecting the cerebral and cerebellar cortex. When one considered the biochemical processes involved in the methylmercury cascade with that of the ADZ cascade, one can see both have an extensive range of neuro-pathological degradationpotential.

\section{Neuropathology of ADZ}

As discussed at the beginning of this work, at a macroscopic level ADZ is characterized by loss of neurons and synapses in the cerebral cortex and certain subcortical regions which ultimately results in gross atrophy of the affected region/s. This atrophy can and does involve degeneration in the temporal lobe and parietal lobe, and parts of the frontal cortex and cingulate gyrus [41,42]. At a microscopic level, both amyloid plaques and neurofibrillary tangles are clearly visible with plaques being dense and mostly insoluble deposits of protein and cellular material outside and around neurons, whilst the tangles are insoluble twisted fibers that build up inside the nerve cell $[41,42]$. Characteristically in older individuals, plaques and tangles occur over time. However, in the brains of ADZ patients, they are at a much greater level and at differing locations throughout the brain.

\section{Glucose Consumption and ADZ}

Due to the brain being extremely metabolically active, it metabolizes a large amount of glucose to produce cellular energy in the form of adenosine triphosphate (ATP). The human brain, although requiring very high energy demands, is not openly available to utilize substrates for energy production as it relies almost entirely on circulating glucose for its energy needs $[43,44]$. Due to the brain having a high dependence on glucose, the risk is that if glucose distribution is disrupted, then the brain will not be able to produce ATP ultimately leading to structural changes due to synapse and cell dysfunction [45,46]. Several authors [47-49]. have observed the decreased utilization of glucose in the brains of ADZ patients early in the disease, before clinical signs of cognitive impairment occur.

It has been noted that the decrease in glucose metabolism worsens as clinical symptoms develop and the disease progresses $[47,48]$. Research has found that a $17 \%-24 \%$ decline in cerebral glucose metabolism has been noted in patients with ADZ [50,51]. Low glucose and its impact upon cerebral glucose metabolism ultimately forms a pattern as seen in the ADZ brain. Anatomically, it has been suggested that the posterior cingulate, parietal, temporal and prefrontal cortices are the most affected areas of cerebral glucose decrease. It is understood that these areas are responsible for multiple aspects of memory and cognition and it has been further suggested that this unique metabolic pattern is reproducible and even a diagnostic tool for ADZ [50,51].

\section{In Conclusion}

It is no new theory that there is a link between disease and diet and indeed that diet and health go hand in hand with one another. Diet and the links it has within many of the major diseases affecting the human population today is very much at the forefront of modern medical science. The United States has the highest mortality rates among diseases that have a dietary link or root cause. This work although only extremely brief has explored a few such considerations such as the link between nutritional intake and the dementias, namely ADZ. As considered in this work, the link between decreased protein synthesis and the correlated degradation upon brain tissue has many facets that need to be considered in relation to ADZ. It is understood that a lack of protein synthesis proportionally decreases normal biochemical and neuro-physiological processing. This point has a plethora of additional considerations. Many patients with forms of dementia are classically malnourished, be this due to the condition itself and or to pre-existing health issues. If protein synthesis is a key area of consideration in both pre and post ADZ manifestation, then should maximal protein and nutritional absorption be the primary focus of research when considering dietary input in ADZ progression? And are patients/families with ADZ or indeed patients/families that may be in risk groups, receiving adequate dietary input information? The work of Morris et al. considered the link of mercury within a dietary context namely fish and shellfish. Interestingly, it has long been understood that mercury is more prevalent within the above food groups. Fish and shellfish are an excellent source of rich protein and as mentioned, protein synthesis is a key point of consideration within dementia's pathological trademark. Does the increased protein absorption further drive protein synthesis regardless of the increased level risk of methylmercury exposure? And does this increased mercury 


\section{Anatomy Physiology \& biochemistry international journal}

level play a significant enough role in ADZ progression to override protein-dietary value?

The risks of mercury are without doubt there, and indeed they are very real to the unborn and developing fetus. But how marked and how relevant are the levels of mercury in diets rich in fish and shellfish and are the risks of mercury exposure less significant than the risks of protein and nutrient malnourishment? The problem may be self-propagating as the patient who has ADZ may become increasingly malnourished as the disease progresses. This phase of malnourishment may then further drive disease progression at a faster rate. This is of course speculative and would require further investigation. However, there is strong evidence showing that diet and the disease progression are linked. Much like wound healing times in malnourished post-operative patients, can the same principal be considered when thinking about the progressive nature of ADZ? Is the correlative factor for demographics more to do with key dietary points and is this what is making countries such as the United States at much greater risk of ADZ?

A further point of dietary consideration is that of the role of glucose. As discussed, the brain is extremely metabolically active and therefore metabolizes large amounts of glucose to produce cellular energy in the form of adenosine triphosphate (ATP). The brain which has a high dependence on circulating glucose is therefore at extreme risk if it becomes less available which ultimately results in loss of ATP. Research has found that there is decreased utilization of glucose in the brains of ADZ patients early in the disease, before clinical signs of cognitive impairment occur. It has been noted that the decrease in glucose metabolism worsens as clinical symptoms develop and the disease progresses. When one considers the dietary link between glucose and type 2 diabetes, it is easy to see why the United States has much higher prevalence than other parts of the world due to the availability of high fat high sugar products at low cost. Can this cultural norm of poor dietary intake also be the predisposing factor-instigator for ADZ progression across the Unites States?

As briefly discussed, The Tau Hypothesis (Th) proposes that the tau protein abnormalities initiates ADZ disease progression via hyper-phosphorylation of the tau protein with other threads of tau culminating with the formation of neurofibrillary tangles inside nerve cell bodies resulting in the destruction of the microtubules leading to the destruction of the cell's cytoskeleton thus forming the base dis-configuration as seen in ADZ. The hypothesis suggest that it is this abnormal protein accumulation which ultimately causes the classic manifestation of ADZ. The exact reason for the abnormal nature of the protein and what the link is with diet is certainly an area that could be further explored. As mentioned earlier, although at this point science is unable to untangle the steps, it may be that at some future point the predisposing step will be noted and or linked to a root cause/s which will in turn allow for implemental steps to be addressed to manage such biochemical crises. The vascular hypothesis although different from the tau hypothesis does have a thread of commonality in relation to that of diet and supplementation. As with many diseases that alter the functioning of the vascular system, the impact of the western diet culture has seen vascular disease mortality rates sky rocket. The overall function of the vascular system is to perfuse blood to all regions and organs of the body. This has become compromised due to diseases such as coronary artery diseases etc. Any detrimental effect upon the vascular system will result in overall body systems not becoming adequately perfused, thus causing cellular death over time. This is no different with the brain and brain tissue. Inadequate cerebral vascular distribution will ultimately alter neurological functioning as seen with ADZ.

So why if there is link between protein synthesis and ADZ does mercury and heavy metal toxicity matter? Heavy metal toxicity has been shown to have neurodegenerative properties and indeed if left untreated or unmanaged will cause irreversible damage to human brain tissue. This is especially true in that of the developing fetus. In adults, medicallymanaged chelation relies upon removing heavy metals from the body with one of the major side effects being that of non-specific binding, in which agents bind even essential trace metals in the body, such as copper, zinc and calcium.

This overall mass removal of trace metals can have profound future consequences upon body systems that use these metals in biochemical pathways. Dependent upon the level of metal toxicity, one may be able to consider if pharmacological intervention is the best methodology of treatment. Certainly, in acute poisoning the only method is quick and effective removal with the unfortunate side effect of non-specific binding. Could it be that when we consider ADZ and patients who may have increased metal toxicity that a slow and gradual metal toxicity chelation therapy may be the way of avoiding non-specific binding, whilst also increasing the stress load upon brain tissue caused by the metal toxicity crisis?

\section{In Summation}

The level of ADZ across the United States is at an all-time high. Medical research is looking to manage and indeed cure the disease, but is it looking at managing and preventing the potential instigators? Should future research be far more involved with education of dietary links-cues and should testing for heavy metals be more routinely carried out and available for patients? Could routine screenings for certain age groups and groups in certain dietary risk factors be made more available and would this along with dietary and lifestyle changes further help prevent, reduce or even stop the incidences of ADZ progression? All such questions are of course speculative, and it is not the purpose of this work to dismiss the importance of pharmacological interventions by any means, but rather to enhance the potential for therapeutic outcomes that could correspond with one another. Managing diseases with medication alone has been proven to be a road to 
failure. The management of disease relies upon understanding (patient education), lifestyle choices (diet, exercise etc.), medication and indeed a multi-disciplinary medical approach.

\section{References}

1. CDC (2017) Centre for Disease Control and Prevention. Alzheimer's Disease Mortality by State 2016.

2. Alzheimer's Disease International (2017) World Alzheimer Reports UK.

3. Mutter J, Curth A, Naumann J, Deth R, Walach H (2010) Does Inorganic Mercury Play a Role in Alzheimer's Disease? A Systematic Review and an Integrated Molecular Mechanism. J Alzheimers Dis 22(2): 357-374.

4. Boyd HE (2007) The relationship of the toxic effects of mercury to exacerbation of the medical condition classified as Alzheimer's disease. B.E. Haley/MedicalVeritas 4: 1510-1524.

5. Khatoon S, Campbell SR, Haley BE, Slevin JT (1989) Aberrant guanosine triphosphate $\beta$-tubulin interaction in Alzheimer's Disease. Annals of Neurology 26(2): 210-215.

6. David S, Shoemaker M, Haley B (1998) Abnormal properties of creatine kinase in Alzheimer's Disease brain: correlation of reduced enzyme activity and active site photolabeling with aberrant cytosol-membrane partitioning. Brain Res Mol Brain Res 54(2): 276-287.

7. Hock C, Drasch G, Golombowski S, Müller Spahn F, Willershausen Zönnchen BS, et al. (1998) Increased blood mercury levels in patients with Alzheimer's disease. J Neural Transm 105(1): 59-68.

8. Kosnett MJ (2010) Chelation for heavy metals (arsenic, lead, and mercury): protective or perilous?. Clin Pharmcol Therapeutics 88(3): 412-415.

9. Howland MA, Nelson LS, Lewin NA, Howland MA, Hoffman RS, et al. (2011) Antidotes in Depth (A27): Succimer (2,3-Dimercaptosuccinic Acid). McGraw-Hill pp.1284-1289.

10. Heather C Hatcher, Ravi N Singh, Frank M Tortim, Suzy V Torti (2009) Synthetic and natural iron chelators: therapeutic potential and clinical use. Future Med Chem 1(9): 1643-1670.

11. Queen HL (1998) Chronic Mercury Toxicity: New Hope Against an Endemic Disease, Queen and Company Health Communications. Colorado Springs, Colorado.

12. Renzoni A, Zino F, Franchi E (1998) Mercury Levels Along the Food Chain and Risk for Exposed Populations. Environmental Research 77(2): 68-72.

13. Drexler H, Schaller KH (1998). The Mercury Concentration in Breast Milk Resulting from Amalgam Fillings and Dietary Habits. Environmental Research 77(2): 124-129.

14. DeWayne Ashmead H (2012) Amino Acid Chelation in Human and Animal Nutrition. CRC Press pp. 269.

15. David Rakel (2017) Integrative Medicine. Elsevier Science Publishing pp.1152.

16. Roller S (2003) Natural Antimicrobials for the Minimal Processing of Foods. Woodhead Publishing Limited pp. 320.

17. Freedland R (1977) A Biochemical Approach to Nutrition. Chapman and Hall London.

18. World Health Rankings (2018) Rates of Alzheimer's Deaths Per country.

19. Marina Ramirez-Alvarado, Jeffery W. Kelly, Christopher M. Dobson (2010) Protein Misfolding Diseases: Current and Emerging Principles and Therapies. Wiley and Sons Publishing pp.1102.
20. Ghiso Jorge A, Rostagno Agueda A (2017) Protein Folding Disorders of The Central Nervous System. World Scientific Publishing pp. 336.

21. Vladimir Uversky, Yuri Lyubchenko (2013) Bio-nanoimaging: Protein Misfolding and Aggregation. Elsevier Science Publishing USA, pp. 552.

22. Martha Clare Morris ScD, John Brockman, Julie A Schneider, MPH Yamin Wang, et al. (2016) Association of Seafood Consumption, Brain Mercury Level, and APOE $\varepsilon 4$ Status with Brain Neuropathology in Older Adults. JAMA 315(5): 489-497.

23. Fung YK, Meade AG, Rack EP, Blotcky AJ (1997) Brain mercury in neurodegenerative disorders. J Toxicol Clin Toxicol 35(1): 49-54.

24. Frederic P Miller, Agnes F Vandome, John Mc Brewster (2010) Alzheimer's Disease: Biochemistry of Alzheimer's Disease, Caregiving and Dementia, Alzheimer's Disease Clinical Research, Long-term Memory, Immunotherapy. Alpha-Script Publishing USA p. 72.

25.J Robin Harris, Falk Fahrenholz (2005) Alzheimer's Disease: Cellular and Molecular Aspects of Amyloid beta. Springer Publishing, Germany.

26. Colin J Barrow, David H Small (2007) Abeta Peptide and Alzheimer's Disease: Celebrating a Century of Research. Springer Publishing, UK.

27. Marina Ramirez Alvarado, Jeffery W Kelly, Christopher M Dobson (2010) Protein Misfolding Diseases: Current and Emerging Principles and Therapies. Wiley Publications, USA.

28. Pierfausto Seneci (2014) Molecular Targets in Protein Miss-folding and Neurodegenerative Disease. Elsevier Publishing, Italy, pp. 314.

29. Ronald Ross Watson, Victor R Preedy (2008) Botanical Medicine in Clinical Practice. Trove Wallingford.

30. Dennis J Mckenna, Kenneth Jones, Kerry Hughes, Virginia M Tyler (2002) Botanical Medicines: The Desk Reference for Major Herbal Supplements, Second Edition Taylor and Francis, UK pp. 1168.

31. Iris F F. Benzie, Sissi Wachtel Galor (2011) Herbal Medicine: Biomolecular and Clinical Aspects, Second Edition. Taylor and Francis, USA.

32. Colin R Martin, Victor R Preedy (2015) Diet and Nutrition in Dementia and Cognitive Decline. Elsevier Publishing UK pp. 1260.

33. Clarkson TW, Magos L, Myers GJ (2003) The toxicology of mercury - current exposures and clinical manifestations. New Engl J Med 349(18): 1731-1737.

34. Clarkson TW (2002) The three modern faces of mercury. Environmental Health Perspective 110 Suppl 1:11-23.

35. World Health Organization (2007) Exposure to Mercury: A Major Public Health Concern - Preventing Disease Through Healthy Environment, World Health Organization, Geneva.

36. J Robin Harris, Falk Fahrenholz (2005) Alzheimer's Disease: Cellular and Molecular Aspects of Amyloid beta. Springer Publishing Germany.

37. Colin J Barrow, David H Small (2007) Abeta Peptide and Alzheimer's Disease: Celebrating a Century of Research. Springer Publishing London.

38. Sangram S Sisodia, Rudolph E Tanzi (2007) Alzheimer's Disease: Advances in Genetics, Molecular and Cellular Biology. Springer Publishing Chicago.

39. Yeates KO, Mortensen ME (1994) Acute and chronic neuropsychological consequences of mercury vapour poisoning in two early adolescents. J Clin Exp Neuropsychol 16(2): 209-222.

40. Austin D (2008) An epidemiological analysis of the 'autism as mercury poisoning' hypothesis. Int J Risk Safety Med 20: 135-142.

41. Sontag E, Nunbhakdi Craig V, Sontag JM, Diaz Arrastia R, Ogris E, et al. (2007) Protein phosphatase 2A methyltransferase links homocysteine 
metabolis with tau and amyloid precursor protein regulation. J Neurosci 27(11): 2751-2759.

42. Sontag E, Hladik C, Montgomery L, Luangpirom A, Mudrak I, et al. (2004) Downregulation of protein phosphatase 2A carboxyl methylation and methyltransferase may contribute to Alzheimer disease pathogenesis. J Neuropathol Exp Neurol 63(10): 1080-1091.

43. Vafai SB, Stock JB (2002) Protein phosphatase 2A methylation: a link between elevated plasma homocysteine and Alzheimer's disease. FEBS Lett 51: 1-4.

44. Mulder C1, Schoonenboom NS, Jansen EE, Verhoeven NM, van Kamp GJ et al (2005) The transmethylation cycle in the brain of Alzheimer patients. Neurosci Lett 386(2): 69-71.

45. Linnebank M, Linnebank A, Jeub M, Klockgether T, Wullner U, et al. (2004) Lack of genetic dispositions to hyperhomocysteinemia in Alzheimer disease. Am J Med Gen Part A 131(1): 101-102.

46. Beyer K, Lao JL, Latorre P, Ariza A (2005) Age at onset: an essential variable for the definition of genetic risk factors for sporadic Alzheimer's disease. Ann N Y Acad Sci 1057: 260-278.
47. Beyer K, Lao JI, Latorre P, Riutort N, Matute B, et al. (2003) Methionin synthase polymorphism is a risk factor for Alzheimer disease. Neuroreport 14(10): 1391-1394.

48. Stanger O, Fowler B, Piertzik K, Huemer M, Haschke Becher E, et al. (2009) Homocysteine, folate and vitamin B12 in neuropsychiatric diseases: review and treatment recommendations. Expert Rev Neurother 9(9): 1393-1412.

49. Lars Björkman, Birgitte F Lundekvam, Torgils Lægreid, Bjørn I Bertelsen, et al. (2007) Mercury in human brain, blood, muscle and toenails in relation to exposure: an autopsy study. Environ Health 6: 30

50. Oulhaj A, Refsum H, Beaumont H, Williams J et al (2009) Homocysteine as a predictor of cognitive decline in Alzheimer's disease. Int J Ger Psychiatry 25(1): 82-90.

51. Durakoglugil MS, Chen Y, White CL, Kavalali ET, Herz J (2009) Reelin signaling antagonizes beta-amyloid at the synapse. Proc Nat Acad Sci 106(7): 15938-15943. 